\title{
Role of Diagnostic Laparoscopy in Nonspecific Chronic Pain Abdomen
}

\author{
Subhash $^{1}$, Tanweer Karim² ${ }^{2}$ Atul Jain ${ }^{3}$, Nabal K Mishra $^{4}$, Gaurav Patel ${ }^{5}$, Subhajeet Dey ${ }^{6}$
}

\begin{abstract}
Introduction: Laparoscopy has established its role (diagnostic as well as therapeutic role) in patients with nonspecific chronic pain abdomen. In case of diagnostic dilemma and uncertainty, use of laparoscopy can help to avoid unnecessary laparotomy, provides accurate diagnosis, and helps to plan for surgical intervention if required. However, the role of laparoscopy in nonspecific chronic pain abdomen is still debated.

Aims and objectives: To assess the accuracy of laparoscopy in the diagnosis of nonspecific chronic pain abdomen and its ability to avoid unnecessary exploratory laparotomy with complications and limitations associated with laparoscopy including failure rate.

Materials and methods: This prospective descriptive study was conducted for a period of 1 year in patients with nonspecific chronic pain abdomen for more than 3 months attending the outpatient department or emergency department when clinical features and investigations are not conclusive.

Results: Sixty-two patients in age-group from 15 to 60 years were studied. Overall $85.48 \%$ of patients had resolution of pain after diagnostic laparoscopy with diagnostic accuracy in our study of $88.7 \%$.

Conclusion: Diagnostic laparoscopy should be considered as one of the gold standard tests for diagnosing the nonspecific chronic pain abdomen, when noninvasive diagnostic modality failed in diagnosing cause.

Keywords: Diagnostic laparoscopy, Nonspecific chronic pain abdomen, NCPA, chronic pelvic pain.

World Journal of Laparoscopic Surgery (2021): 10.5005/jp-journals-10033-1464
\end{abstract}

\section{INTRODUCTION}

Nonspecific chronic abdominal pain is a diagnostic challenge for a clinician. These patients generally visit different physicians and many investigations are done for reaching the diagnosis in this process. Chronic abdominal pain without any specific etiological diagnosis at the end of diagnostic workup for more than 3 months is called as nonspecific chronic pain abdomen (NCPA). ${ }^{1-3}$ It can lead to poor quality of life with appearance of depressive symptoms with time. ${ }^{4}$

Surgical consultation is often sought late after other modalities have failed to reach a conclusion or provide relief in their symptomatology. NCPA is a significant clinical problem which accounts for 13 to $40 \%$ of all surgical admissions and can often lead to repeated laparotomies. ${ }^{5}$ Chronic pelvic pain has a prevalence of $3.8 \%$ in young females and it accounts for $10 \%$ of all outdoor patients visit to gynecologist and $40 \%$ of laparoscopy by gynecologists. $^{6}$

The use of laparoscopy in the diagnosis and management of NCPA has been tried in previous studies. The main aim of laparoscopic evaluation in NCPA is to detect the presence or absence of intra-abdominal organic lesion and also it can diagnose as well as treat different intrabdominal pathologies that are difficult to diagnose by other conventional methods. ${ }^{3}$ It is a safe and effective tool which can establish the etiology and allows for appropriate intervention at the same time or a better planning in such cases. ${ }^{7}$

Adhesions and bands are commonly seen findings, especially in patients with a past history of laparotomy or other abdominal operations. ${ }^{8}$ Other findings such as appendicular pathology, hepatobiliary causes, and endometriosis can be discovered and dealt with laparoscopically. ${ }^{9}$

\begin{abstract}
${ }^{1-6}$ Department of Surgery, ESI PGIMSR and Hospital, Delhi, India
Corresponding Author: Atul Jain, Department of Surgery, ESI PGIMSR and Hospital, Delhi, India, e-mail: docatuljain@gmail.com

How to cite this article: Subhash, Karim T, Jain A, et al. Role of Diagnostic Laparoscopy in Nonspecific Chronic Pain Abdomen. World J Lap Surg 2021;14(2):81-86.
\end{abstract}

Source of support: Nil

Conflict of interest: None

The role of laparoscopy for diagnosis in NCPA is still debated by a number of authors who deny its value in adhesiolysis and consider it controversial and not evidence-based, and therefore, do not recommend it as a part of evaluation and treatment in patients with NCPA. ${ }^{10}$

Although laparoscopy is very frequently used by surgeons in all fields for a wide spectrum of surgical procedures around the globe, its utility for diagnosis in cases of chronic abdominal pain was not favored initially, either due to insufficient data on its effectiveness as a diagnostic modality, lack of training or expertise, or lack of awareness among patients and even doctors. ${ }^{11}$

Diagnostic laparoscopy when compared to open laparotomy is better in terms of visualization and access with minimal trauma except in some retroperitoneal lesions. Laparoscopy also has limitation of tactile sensations and lesions cannot be palpated as compared to open laparotomy. ${ }^{12}$ However, procedure allows quick and thorough inspection of whole peritoneal cavity and pelvic cavity and it is an emerging tool in diagnosis of nonspecific chronic pain abdominal. ${ }^{13}$ 


\section{Aims and Objectives}

The study was done to assess the accuracy of laparoscopy in the diagnosis of NCPA (by comparing its findings with radiological investigations), its ability to avoid unnecessary exploratory laparotomy with complications and limitations associated with laparoscopy including failure rate.

\section{Materials and Methods}

This prospective descriptive study was conducted in a teaching hospital in north India for a period of 1 year. Clinical material for present study comprises the patients with NCPA for more than 3 months attending the outpatient department or emergency department where other clinical symptoms and investigations are not conclusive.

\section{Inclusion Criteria}

- Chronic pain abdomen of more than 3 months of uncertain etiology unexplained by clinical symptoms and signs and other investigations including CECT.

- Age-group of 15 to 60 years.

\section{Exclusion Criteria}

- ASA Grade III, ASA Grade IV.

- Uncorrected coagulopathy.

- Pregnancy.

Diagnostic laparoscopy was performed with standard method after proper preanesthetic checkup and wherever biopsy or other surgical intervention (laparoscopic/open) was required, it was done.

\section{Results}

A total of 62 patients, who fulfill the inclusion and exclusion criteria were included in the study.

\section{Age and Sex Distribution}

In our study, youngest patient was 15 years and oldest was 60 years. The mean age of presentation was 37.37 (Table 1). There was predominance of female gender, who were $34(54.8 \%)$ in comparison to male gender 28 (45.2\%).

Duration of pain before diagnostic laparoscopy: patients with duration of 3 or more months of NCPA were included. Mean duration was 4.6 months. (Fig. 1).

The final diagnosis reached in our study is shown in Table 2 showing the most common cause was recurrent appendicitis (32.2\%).

In our total 62 cases, 54 (87.1\%) cases were diagnosed by laparoscopic procedure (i.e., radiological given diagnosis totally different from laparoscopic findings), rest 8 (12.9\%) cases were radiological as well as laparoscopically same diagnosis.

\section{Conversion and Complications}

In total 62 cases, 50 (80.6\%) cases were treated completely with laparoscopic approach and in remaining 12 (19.4\%) cases, laparoscopy was converted to laparotomy. In these 12 patients, 5 patients underwent lap-assisted right hemicolectomy [HPE-1 mucormycosis (Fig. 2), 3 adenocarcinoma of intestine, 1 diverticulitis], 2 patients had resection of stricture followed by endto-end anastomosis, HPE revealed adenocarcinoma of intestine with negative resected margins, 2 radical cholecystectomy for
Table 1: Age distribution of patients with nonspecific chronic pain abdomen (NCPA)

\begin{tabular}{llc}
\hline Age-group (years) & Number of patients & Percentage (\%) \\
\hline $15-30$ years & 18 & 29.0 \\
$31-45$ years & 28 & 45.2 \\
$46-60$ years & 16 & 25.8 \\
Total & 62 & \multicolumn{2}{c}{100.0} \\
Mean & \multicolumn{2}{c}{37.37} \\
Min-max & \multicolumn{2}{c}{$15-60$} \\
\hline
\end{tabular}

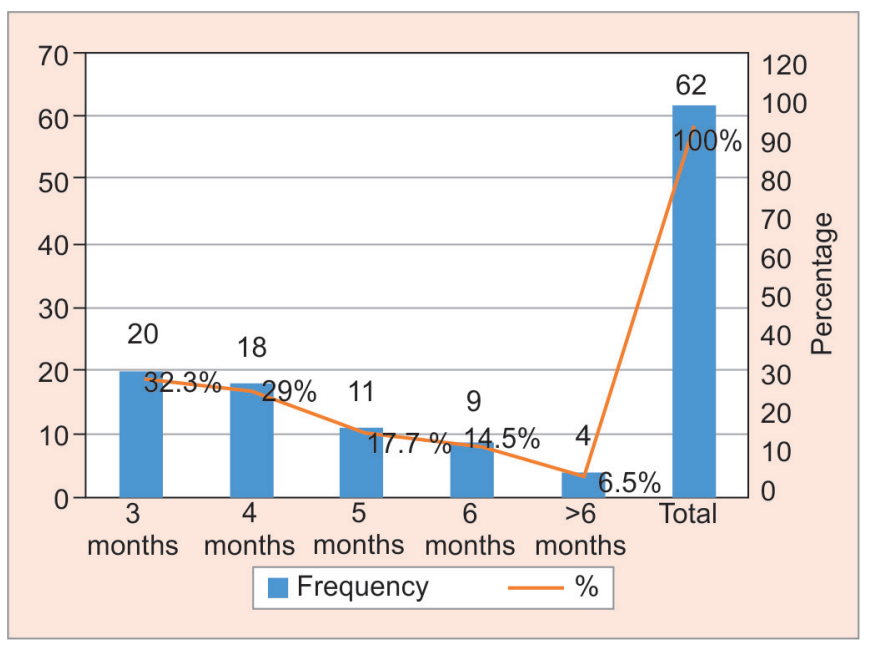

Fig. 1: Showing duration of pain abdomen before diagnostic laparoscopy

Table 2: Frequency and percentage distribution of patients according to final diagnosis

\begin{tabular}{lcc}
\hline Final diagnosis & Frequency & Percentage (\%) \\
\hline Appendicitis (recurrent appendicitis) & 20 & 32.25 \\
Normal study & 7 & 11.29 \\
Reactive mesenteric lymphadenopathy & 6 & 9.67 \\
CA intestine & 5 & 8.06 \\
Bands and adhesions & 4 & 6.45 \\
Chronic cholecystitis & 3 & 4.83 \\
TB of IC junction & 3 & 4.83 \\
Normal appendix & 1 & 1.61 \\
Gallbladder carcinoma & 2 & 3.22 \\
Diverticulitis & 1 & 1.61 \\
Granulomatous pancreatitis & 1 & 1.61 \\
Granuloma of parietal wall & 1 & 1.61 \\
Normal gallbladder & 1 & 1.61 \\
Pancreatic tuberculosis & 1 & 1.61 \\
Adrenal lipoma & 1 & 1.61 \\
IC junction intususception & 1 & 1.61 \\
Mucormycosis & 1 & 1.61 \\
Mesenteric cyst & 1 & 1.61 \\
Renal cyst & 1 & 1.61 \\
Ovarian cyst & 1 & 1.61 \\
Total & 62 & 100 \\
\hline & &
\end{tabular}


carcinoma of gall bladder, 1 open cholecystectomy for sealed gall bladder perforation. One Whipple's procedure for bulky head of pancreas histopathology (HPE) revealed as granulomatous pancreatitis (Fig. 3), and one open adrenal lipoma excision. There are no postprocedure complications in all 62 cases.

Table 3 shows sensitivity, specificity, and accuracy along with $p$ value significance level of radiological investigations, which was compared with final diagnosis for diagnosing of NCPA. Sensitivity-16.4\%, specificity-57.1\%, and accuracy-21.0\%, $p$-value is 0.125 ( $p$-value significance level is $<0.05$ ).

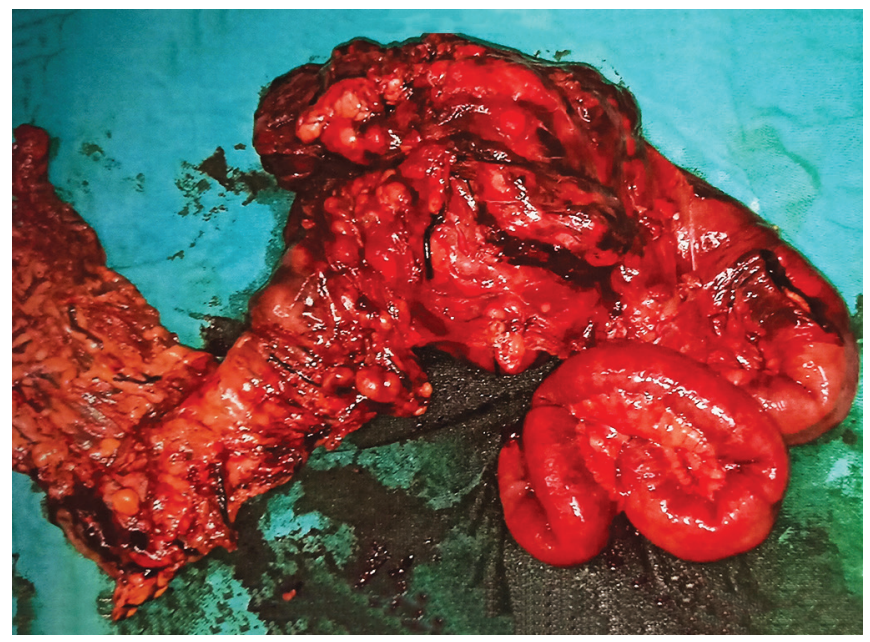

Fig. 2: Intestinal mucormycosis specimen

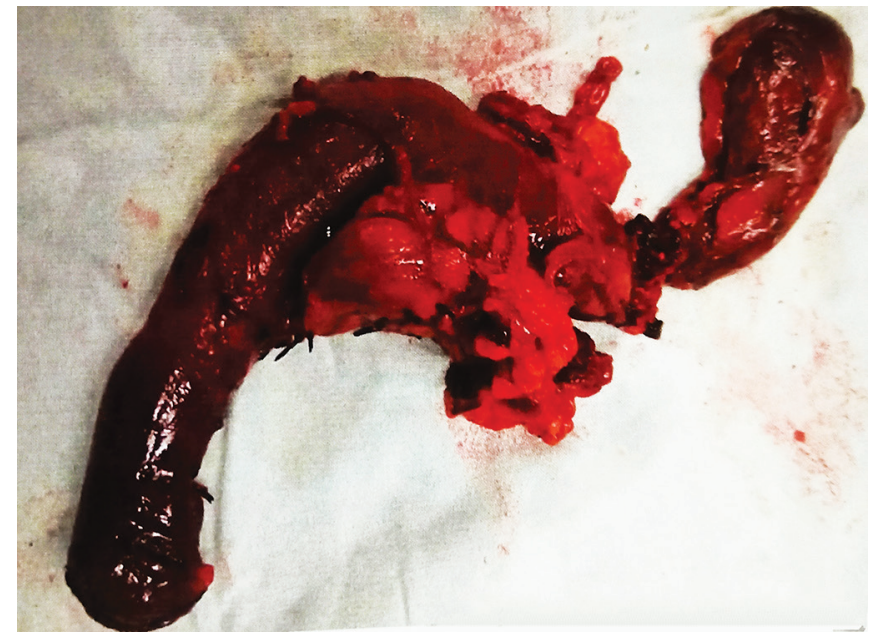

Fig. 3: Whipple's procedure specimen—granulomatous pancreatitis
Table 4 shows sensitivity, specificity, and accuracy along with $p$ value significance level of laparoscopic findings which was compared with final diagnosis in diagnosing of NCPA, sensitivity-98.2\%, specificity-100.0\%, and accuracy-98.4\%, $p$-value is $<0.001$ ( $p$-value significance level is $<0.05$ ).

Table 5 shows radiological findings sensitivity, specificity and accuracy along with $p$ value significance level which was compared with laparoscopic findings for diagnosing of NCPA, sensitivity $-16.7 \%$, specificity-62.5\%, and accuracy-22.6\%, $p$-value is 0.177 ( $p$-value significance level is $<0.05$ ).

\section{Effect of Diagnostic Laparoscopy on Diagnosis}

In this study of 62 cases, in 45 (72.58\%) cases, the final diagnosis was same as that of diagnostic laparoscopy, in $6(9.6 \%)$ cases, the final diagnosis was made after HPE, in 7 cases, the diagnostic laparoscopy (DL) failed to diagnose the cause (normal findings), and in 4 cases, the final and laparoscopic diagnosis were both different (Table 6).

\section{Discussion}

NCPA is a frequent problem, dealt with by different medical specialists. Even after an extensive workup in some patients, no specific cause or pathological condition is found by use of noninvasive investigation, and the pain is often attributed to unsubstantiated diagnosis. Despite of advanced diagnostic machinery with sophisticated methodology to image abdominal contents, establishment of a diagnosis prior to surgery remains difficult for several conditions. Unnecessary or negative laparotomy is painful, increases hospital stay, increases hospital cost, and is associated with a morbidity of 5 to $20 \%$.

Overall in 62 patients, radiological imaging modality gave probable diagnosis in $28(45.16 \%)$ patients, normal study in 10 (16.12\%) patients, and in remaining 24 (38.70\%) patients, diagnosis was inconclusive. Subsequent DL and HPE reveled that out of these 24 cases, 15 (62.5\%) patients are having appendicitis. Based on USG findings, 11 patients were diagnosed as having ileocecal thickening (Fig. 4) and mesenteric lymphadenopathy with tuberculosis (TB) as first differential diagnosis. However, CECT confirmed ileocecal tuberculosis in six cases only. All of them have undergone colonoscopy and biopsy. Colonoscopy biopsy failed to prove tuberculosis in any one of them. Only one of these six cases was finally diagnosed as having ileocecal tuberculosis on HPE following resection of affected segments. Therefore recurrent vague pain in lower abdomen with nonspecific radiological finding may be consistent feature of recurrent or chronic appendicitis and DL seems to be more useful.

In a similar study on 88 patients by Ahmad et al., ${ }^{14} 38$ (43.10\%) patients' abdominal ultrasound was normal. The most common finding noted on USG abdomen and pelvis was

Table 3: Radiological findings compared with final diagnosis

\begin{tabular}{|c|c|c|c|c|c|}
\hline \multirow[b]{3}{*}{ Radiological findings } & \multicolumn{4}{|c|}{ Final diagnosis } & \multirow[b]{3}{*}{$p$ value } \\
\hline & \multicolumn{2}{|c|}{ Positive } & \multicolumn{2}{|c|}{ Negative } & \\
\hline & Frequency & Percentage (\%) & Frequency & Percentage (\%) & \\
\hline Positive & 9 & 16.4 & 3 & 42.9 & \\
\hline Negative & 46 & 83.6 & 4 & 57.1 & 0.125 \\
\hline Total & 55 & 100 & 7 & 100 & \\
\hline Sensitivity & Specificity & PPV & NPV & Accuracy & \\
\hline $16.4 \%$ & $57.1 \%$ & $75.0 \%$ & $8.0 \%$ & $21.0 \%$ & \\
\hline
\end{tabular}


Diagnostic Lap in NCPA

Table 4: Laparoscopic findings compared with final diagnosis

\begin{tabular}{llcllll}
\hline & \multicolumn{4}{c}{ Final diagnosis } & \\
\cline { 2 - 5 } & \multicolumn{3}{c}{ Positive } & \multicolumn{2}{c}{ Negative } & \\
\cline { 2 - 5 } Laparoscopic findings & Frequency & Percentage (\%) & Frequency & Percentage (\%) & p value \\
\hline Positive & 54 & 98.2 & 0 & 0.0 & \\
Negative & 1 & 1.8 & 7 & 100.0 & $<0.001$ \\
Total & 55 & 100 & 7 & 100 & \\
Sensitivity & Specificity & PPV & NPV & Accuracy & \\
$98.2 \%$ & $100.0 \%$ & $100.0 \%$ & $87.5 \%$ & $98.4 \%$ & \\
\hline
\end{tabular}

Table 5: Radiological findings compared with laparoscopic findings

\begin{tabular}{|c|c|c|c|c|c|}
\hline \multirow[b]{3}{*}{ Radiological findings } & \multicolumn{4}{|c|}{ Laparoscopic findings } & \multirow[b]{3}{*}{$p$ value } \\
\hline & \multicolumn{2}{|c|}{ Positive } & \multicolumn{2}{|c|}{ Negative } & \\
\hline & Frequency & Percentage (\%) & Frequency & Percentage (\%) & \\
\hline Positive & 9 & 16.7 & 3 & 37.5 & \\
\hline Negative & 45 & 83.3 & 5 & 62.5 & 0.177 \\
\hline Total & 54 & 100 & 8 & 100 & \\
\hline Sensitivity & Specificity & PPV & NPV & Accuracy & \\
\hline $16.7 \%$ & $62.5 \%$ & $75.0 \%$ & $10.0 \%$ & $22.6 \%$ & \\
\hline
\end{tabular}

Table 6: Effect of diagnostic laparoscopy on diagnosis

\begin{tabular}{lcc}
\hline Diagnosis status & Frequency & Percentage (\%) \\
\hline Confirmed diagnosis & 45 & 72.58 \\
Failed in diagnosing & 7 & 11.29 \\
Diagnosed after laparoscopy & 6 & 9.67 \\
Changed diagnosis & 4 & 6.45 \\
Total & 62 & 100 \\
\hline
\end{tabular}

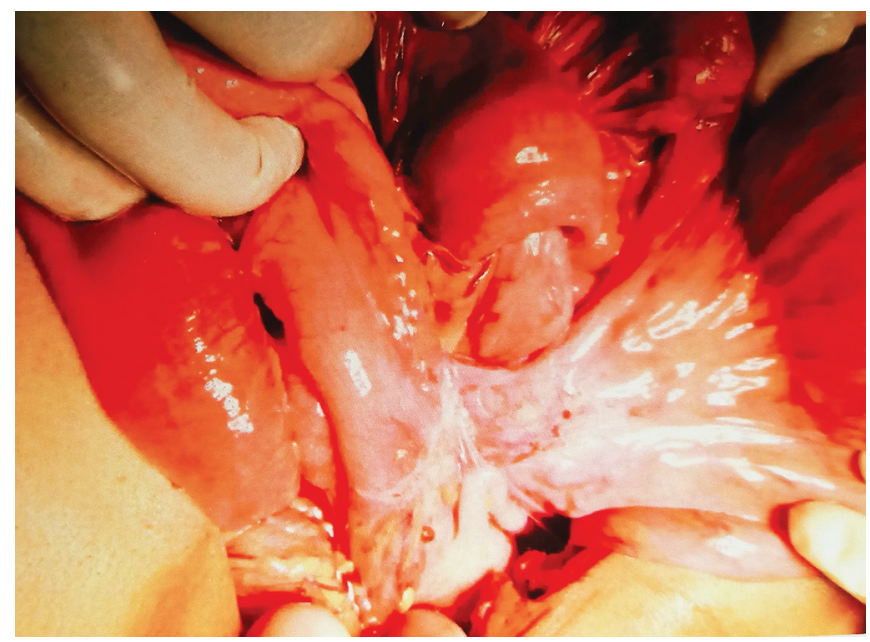

Fig. 4: Ileocecal mass

distended bowel loops in RIF. Benign hypertrophy of prostate was reported in two patients. USG pelvis in 51 of 59 patients was normal. In the remaining patients, minimal free fluid in cul-de-sac was reported. All subjects underwent CT scanning, out of which, $63(52.5 \%)$ patients had a change in findings when compared with the findings on USG. The CT scan was better able to suggest dilation of gut loops and reteroperitoneal/mesentric
Table 7: Comparison of diagnostic accuracy of laparoscopy in various study

\begin{tabular}{ll}
\hline Study & Diagnostic accuracy (\%) \\
\hline Mehta's et al. & 88 \\
Al-Akeely et al. & 94 \\
El-Labban et al. & 83.3 \\
Ahmad et al. & 85.2 \\
Present study & 88.7 \\
\hline
\end{tabular}

lymphadenopathy. Twenty-four subjects out of 88 cases (24.2\%) had altogether new findings, while 64 (72.4\%) cases had findings like the radiological means. Twenty-five out of 64 had new finding along with previous finding. Therefore, DL enables the surgeon to visualize surface anatomy of intra-abdominal organs with greater details better than any other imaging modality.

DL has been used as a diagnostic tool in patients in nonspecific pain abdomen in numerous studies. However, it is still not a standard of care for this subset of patients. In our series, 62 (100\%) patients of NCPA were subjected to DL and reached to final definitive diagnosis in 55 (88.7\%) patients, and in 7 (11.3\%) patients, we could not reach to any diagnosis (i.e., normal study). DL could establish diagnosis in $88 \%$ cases in Mehta's series, ${ }^{15}$ whereas in the series of Al-Akeely et al., ${ }^{16}$ it was $94 \%$. Our series had a diagnostic accuracy of $88.7 \%$. Ahmad et al. ${ }^{14}$ could reach to final diagnosis in 75 of 88 cases of NCPA after DL. This is like the study carried by Labban and Hokkam et al. ${ }^{17}$ in which DL provided a definitive diagnosis in 25 (83.3\%) of the 30 cases of NCPA.

\section{Diagnostic Accuracy}

DL was able to establish diagnosis in $88 \%$ of cases in Mehta's series, whereas in the series of Al-Akeely, it was $94 \%$. Our series had a diagnostic accuracy of $88.7 \%$ (Table 7 ).

In this study, appendicitis (recurrent appendicitis) was most common diagnosis, seen in 20 (32.25\%) patients, and laparoscopic 
appendectomy was done in all of them. In a study by Ahmad et al., appendicular pathology was present in $32.9 \%$ of cases and in a study by Ates et al., $47.2 \%$ of patients were diagnosed with appendicitis. ${ }^{18}$

In a study by Fayez et al., ${ }^{19}$ records of chronic abdominal pain undergoing appendectomy were reviewed; $92 \%$ of patient's appendices had abnormal histological findings and the $95 \%$ of patients had resolution of pain. Raymond et al. ${ }^{20}$ reported improvement of pain in $74 \%$ of patients with chronic right lower abdominal pain.

In our study, out of 62 patients, 21 (33.87\%) patients who underwent appendectomy for NCPA, in that 20 (95.23\%) patient's HPE revealed as recurrent appendicitis, in these patient's pain was resolved, one (4.76\%) patient HPE revealed as normal appendix; in this patient, pain was not resolved. So our study also correlating with study by Fayez et al. and Raymond et al. in alleviating pain in patients with NCPA.

For comparison of therapeutic efficiency of laparoscopy, Raymond et al. study reported that more than $70 \%$ of patients had long term pain relief and Paajanen et al. in their study reported that laparoscopy alleviates the symptoms in more than $70 \%$ of patients. In our study out of 62 patients, 53 (85.48\%) patients had pain relief after $D L$. Remaining nine patient pain was not relieved (in that seven patient's final diagnosis revealed as normal study, one patient appendicular pathology revealed normal, another one patient diagnosed case of abdominal tuberculosis). These two studies correlate well with our study and it should be considered if other diagnostic tests are inconclusive (Table 8).

In a study by Palanivelu et al. ${ }^{21}$ out of 230 patients diagnosed to have abdominal TB by DL, 132 (57.4\%) were males and 98 (42.6\%) females. The peritoneal TB cases were treated by $D L$, peritoneal biopsy, followed by antitubercular treatment. In a study by Rai et al. ${ }^{22}$ on the role of DL on abdominal TB, 36 patients were included in which 24 (66.6\%) patients were male and 12 (33.4\%) female. In our study, four patients diagnosed to have abdominal TB out of which three (4.83\%) are female and one (1.61\%) male. All patients were managed with CAT-1 ATT.

Our study report revealed improvement or resolution of pain abdomen in patients with abdominal tuberculosis is $75 \%$.

Arya and Gaur study revealed out of 49 patients, bands and adhesions in 4 (8.16\%) patients, no organic cause found in 5 (10.20\%) patients, and diverticulitis in 1 (2.04\%) patient. ${ }^{23}$

In our study out of 62 patients, no organic cause found in 7 (11.29\%) patients, diverticulitis in 1 (1.61\%) patient, and 4 (6.45\%) patients had bands and adhesions for that adhesiolysis done as a definitive procedure, and all $4 \%$ patients had resolution of their pain after adhesiolysis. So our study well correlated with Arya et al. study.

Compared to series of Mehta et al. out of 21 patients, 4 who underwent laparotomy, conversion rate was 19\%. In another series, Al-Akeely had $6 \%$ conversion rate.

In our series out of 62 patients, 12 who underwent laparotomy, conversion rate is $19.4 \%$. With the growing availability of trained and experienced laparoscopic surgeons along with improved

Table 8: Comparison of therapeutic efficiency of laparoscopy in various studies

\begin{tabular}{ll}
\hline Study & Therapeutic efficiency (\%) \\
\hline Raymond et al. $^{20}$ & $>70$ \\
Paajanen et al. & \\
Present study & $>70$ \\
\hline
\end{tabular}

machinery, the morbidity of laparoscopy is much less and not, and with improved skills, conversion rates should be low.

\section{Conclusion}

$D L$ should be considered as one of the gold standard test for diagnosing the NCPA, when noninvasive diagnostic modality failed in diagnosing cause. It can prevent the delay in definitive diagnosis and negative laparotomies in these cases. It has diagnostic, therapeutic use and in some cases can have placebo effect as well.

\section{References}

1. Camilleri M. Management of patients with chronic abdominal pain in clinical practice. Neurogastroenterol Motil 2006;18(7):499-506. DOI: 10.1111/j.1365-2982.2005.00744.x.

2. Townsend CO, Sletten CD, Bruce BK, et al. Physical and emotional functioning of adult patients with chronic abdominal pain: comparison with patients with chronic back pain. J Pain 2005;6(2): 75-83. DOI: 10.1016/j.jpain.2004.10.009.

3. Paajanen $\mathrm{H}$, Julkunen $\mathrm{K}$, Waris $\mathrm{H}$. Laparoscopy in chronic abdominal pain. A prospective nonrandomized long-term follow-up study. J Clin Gastroenterol 2005;39(2):110-114. DOI: 10.1097/01. mcg.0000150174.88953.6e.

4. Magni G, Rossi MR, Rigatti-Luchini S, et al. Chronic abdominal pain and depression. Epidemiologic findings in the United States. Hispanic health and nutrition examination survey. Pain 1992;49(1):77-85. DOI: 10.1016/0304-3959(92)90191-D.

5. Irvin TT. Abdominal pain: a surgical audit of 1190 emergency admissions. Br J Surg 1989;76(11):1121-1125. DOI: 10.1002/ bjs.1800761105.

6. Reiter RC. Chronic pelvic pain. Clin Obstet Gynaecol 1990;33(1): 130-136. DOI: 10.1097/00003081-199003000-00018.

7. Onders RP, Mittendorf EA. Utility of laparoscopy in chronic abdominal pain. Surgery 2003;134(4):552-554. DOI: 10.1016/s00396060(03)00277-0.

8. Szomstein S, Lo Menzo E, Simpfendorfer C, et al. Laparoscopic lysis of adhesions. World J Surg 2006;30(4):535-540. DOI: 10.1007/s00268005-7778-0.

9. Salky BA, Edye MB. The role of laparoscopy in the diagnosis and treatment of abdominal pain syndromes. Surg Endosc 1998;12(7): 911-914. DOI: 10.1007/s004649900744.

10. Swank DJ, Swank-Bordewijk SC, Hop WC, et al. Laparoscopic adhesiolysis in patients with chronic abdominal pain: a blinded randomised controlled multi-centre trial. Lancet 2003;361(9365): 1247-1251. DOI: 10.1016/s0140-6736(03)12979-0.

11. Nar AS, Bawa A, Mishra A, et al. Role of diagnostic laparoscopy in chronic abdominal conditions with uncertain diagnosis. Niger J Surg 2014;20(2):75-78. DOI: 10.4103/1117-6806.137301.

12. Perri SG, Altillia F, Pietrangeli $F$, et al. Laproscopy in abdominal emergencies. Indications and limitations. Chir Ital 2002;54(2):165-178.

13. Kerman US, Reddy KR. Diagnostic laparoscopy; an update. Endoscopy 2002;34(2):146-153. DOI: 10.1055/s-2002-19847.

14. Ahmad MM, Dar HM, Waseem M, et al. Role of laparoscopy in nonspecific abdominal pain. Saudi Surg J 2014;2(3):71-74. DOI: 10.4103/2320-3846.147021.

15. Mehta S, Juneja I, Udani D. Role of diagnostic laparoscopy in management of acute abdomen. Int J Surg 2009;20(1).

16. Al-Akeely $\mathrm{MH}$. The impact of elective diagnostic laparoscopy in chronic abdominal disorders. Saudi J Gastroenterol 2006;12(1):27-30. DOI: 10.4103/1319-3767.27741.

17. El-Labban GM, Hokkam EN. The efficacy of laparoscopy in the diagnosis and management of chronic abdominal pain. J Minim Access Surg 2010;6(4):95-99. DOI: 10.4103/0972-9941.72594.

18. Mustafa Ates, Sedat Sevil, Mahmut Bulbul. Routine use of laparoscopy in patients with clinically doubtful diagnosis of appendicitis. 
J Laparoendosc Adv Surg Tech 2008;18(2):189-193. DOI: 10.1089/ lap.2007.0040.

19. Fayez JA, Toy NJ, Flanagan TM. The appendix as the cause of chronic lower abdominal pain. Am J Obstet Gynecol 1995;172:122-123. DOI: 10.1016/0002-9378(95)90097-7.

20. Raymond $\mathrm{P}$, Onders MD, Elizabeth A, et al. Utility of laparoscopy in chronic abdominal pain. Surgery 2003;134(4):549-554. DOI: 10.1016/ s0039-6060(03)00277-0.
21. Palanivelu C. Diagnostic laparoscopy-indications, tuberculosis and adhesiolysis. In: Art of laparoscopic surgery. Textbook and atlas, vol. 1. 2007. p. 153-178.

22. Rai $S$, Thomas WM. Diagnosis of abdominal tuberculosis the importance of laparoscopy. J R Soc Med 2003;96(12):586-588. DOI: 10.1258/jrsm.96.12.586.

23. Arya PK, Gaur KJBS. Laparoscopy, a tool in diagnosis of lower abdominal pain. Indian J Surg 2004;66(4):216-220. 